\title{
CRECIMIENTO HASTA LOS 42 - 44 MESES DE EDAD Y ESTIMACIÓN DE PARÁMETROS GENÉTICOS DE 23 PROCEDENCIAS Y 196 FAMILIAS DE Eucalyptus camaldulensis Denh EN CUATRO SITIOS DE LA ZONA CENTRAL DE CHILE.
}

\author{
CARLOS ALVEAR S. (*) y BRAULIO GUTIÉRREZ C. (**)
}

(*) Médico Veterinario, Doctorado en Ciencias Agrarias. Instituto Forestal (INFOR). Santiago, Chile.

$\left(^{* *}\right)$ Ingeniero Forestal (e). Instituto Forestal (INFOR). Santiago, Chile.

\section{RESUMEN}

Se analiza la variación en crecimiento juvenil y sobrevivencia a los 42 - 44 meses de edad, de 196 familias australianas $y$ dos testigos nacionales de Eucalyptus camaldulensis establecidas en cuatro sitios de la zona central de Chile (32-35 Lat. Sur). Tambien se estiman heredabilidades individuales para las caracteristicas de crecimiento y las correlaciones genéticas y fenotipicas entre estas variables.

Se constata la existencia de diferencias estadisticamente significativas entre procedencias y dentro éstas, confirmándose a algunas familias procedentes de los lagos Hindmarsh $y$ Albacutua, del Estado de Victoria, como las de mayor crecimiento.

Las heredabilidades individuales asociadas a las variables de crecimiento son en general bajas, sugiriendo que la ganancias genéticas que pueden lograrse en base a selección individual y propagación por semillas serán escasas.

Se concluve que un indice de selección aplicado a nivel de familias es la estrategia más adecuada para un programa de mejoramiento genético de la especie, y que la selección debe orientarse principalmente familias a procedentes del estado de Victoria.

Palabras clave: Eucalyptus camaldulensis, Procedencia, Progenies, Crecimiento inicial, Parámetros genéticos. 


\section{ABSTRACT}

The variation in early growth and survival at 42 - 44 months old in 196 australian progenies and two national control-progenies of Eucalyptus camaldulensis established in four sites in the central zone of Chile (32- $35^{\circ}$ South Latitude) is assesed. The individual heritability and both. the genetical and phenotypical correlations between these variables are also estimated.

Statistical differences were found within and between the provenances. Some progenies from Lakes Albacutya and Hindmarsh (Victoria) are confirmed as the highest growths.

The indivual heritabilities linked to the growth variables are low, suggesting a low genetic gain obtainable from individual selection and seed propagation.

The family selection or the selection index will be the most suitable strategy for a genetic improvement program with this specie. This program should consider mainly progenies from Victoria.

Keywords : Eucalyptus camaldulensis, Provenances, Progenies, Early growth, Genetic parameter. 


\section{INTRODUCCIÓN.}

El Instituto Forestal inició en 1962 un amplio programa de introducción de especies forestales, cuyos múltiples ensayos permitieron concluir que Eucalyptus camaldulensis es una de las especies más apropiadas para forestar y reforestar las zonas semiáridas del pais (INFOR, 1986).

Posteriormente, y en base a los resultados de los ensayos anteriores, se establecieron ensayos de procedencias de E. camaldulensis, los que confirmaron la excelente adaptación de esta especie a las zonas semiáridas de Chile y arrojaron los primeros indicios de la gran variabilidad intraespecífica que ella exhibe (Barros, 1990).

Ultimamente, en el marco definido por el proyecto de Mejoramiento Genético del Eucalipto, y considerando que las ganancias genéticas que un programa de este tipo puede generar son consecuencia directa de la amplitud y variabilidad representada en la base genética con que se trabaja (poblaciones bases), el Instituto Forestal procedió a realizar una importación de semillas provenientes de importantes zonas del área de distribución natural de la especie en Australia. Esta semilla después de haber sido viverizada se estableció en cuatro ensayos de procedencias y progenies, cuyo desarrollo y comportamiento se analiza en este documento.

La importancia de contar con una base genética amplia. como la establecida por INFOR, radica en que permite obtener una mayor ganancia genética, además de mantener una población desde la cual seleccionar material para satisfacer las necesidades de un programa de mejoramiento de largo plazo.

Las evaluaciones del crecimiento juvenil en las poblaciones bases son una etapa fundamental en los proyectos de mejoramiento genético. El largo plazo involucrado en estos programas hace deseable que se reduzca el tiempo comprendido entre cada generación de mejoramiento y esto indudablemente se puede conseguir al hacer la selección de los individuos lo más tempranamente posible.

Sin duda que las selecciones tempranas conllevan mucho riesgo. siendo estas más precisas en la medida que los árboles se acercan a la madurez. Aún así, las evaluaciones del crecimiento juvenil resultan imprescindibles para establecer en el futuro la correlación juvenil-adulto, de modo que este seguimiento permita determinar, para cada característica de interés, la edad minima a la que se puede hacer una selección confiable (Infante y Prado, 1989).

Por otra parte. la estimación de parámetros genéticos constituye una invaluable herramienta para la toma de decisiones en el proceso de mejoramiento. La estimación de parámetros como la heredabilidad a lo largo del tiempo. permite determinar el momento en que esta se estabiliza y se hace más eficiente la selección. Además, el contar con una evaluación del grado en que cada caracteristica de interés es transmitida 
desde los progenitores a su descendencia, resulta fundamental para determinar, en conjunto con el diferencial de selección. la ganancia genética asociada a una determinada estrategia de selección.

Por último, la evaluación económica de la ganancia esperada constituirá el parámetro que determine el impacto del programa de mejoramiento.

En virtud de lo anterior, en este documento se analizan las variables de crecimiento en etapa juvenil de procedencias y progenies de E. camaldulensis y se estiman los parámetros genéticos asociados a estas variables.

\section{OBJETIVOS}

Evaluar la variabilidad en crecimiento juvenil de E. camaldulensis e individualizar las procedencias y progenies más adecuadas para maximizar la producción volumétrica en las plantaciones que se establezcan en la zona central de Chile.

Determinar parámetros genéticos para las variables de crecimiento de la especie.

\section{MATERIAL Y MÉTODO}

Para dar cumplimiento a los objetivos enunciados se utilizó la información correspondiente de cuatro ensayos de procedencias y progenies de E. camaldulensis, realizados a edades entre 42 y 44 meses.

Las variables consideradas fucron la sobrevivencia (SOB). altura total (ALT), diámetro de cuello (DAC) cuando las plantas presentaban menos de $1.3 \mathrm{~m}$ de altura y diámetro a la altura del pecho (DAP) cuando las plantas superaban los $1,3 \mathrm{~m}$ de altura. También. se consideró a la variable $D^{2} \mathrm{H}$. por cuanto combina en un solo valor al diámetro y la altura. siendo un buen estimador del crecimiento en volumen (Infante y Prado. 1991).

\section{Antecedentes de la Colección de Semillas.}

La colección de semillas empleada en los ensayos es una muestra que representa algunas zonas del área de distribución natural de la especie, encontrándose caracterizada en el Cuadro $\mathrm{N}^{\circ} 1$. La semilla se encuentra individualizada según lugar de origen (procedencia) y árbol madre (familia). La colección representada en los ensayos incluye 23 procedencias y 196 familias australianas. a las que se agregan como testigos dos procedencias de raza local: La Ligua y Lolol. 


\section{Cuadro $\mathrm{N}^{\mathrm{O}} 1$.}

\section{CARACTERIZACIÓN DE LAS PROCEDENCIAS DE Eucalyptus camaldulensis CONSIDERADAS EN LOS ENSAYOS.}

\begin{tabular}{|c|c|c|c|c|c|c|c|c|c|}
\hline $\begin{array}{l}\text { PRO- } \\
\text { CE- } \\
\text { DEN- } \\
\text { CIA }\end{array}$ & LUGAR DE ORIGEN & (1) & $\mathrm{N}^{\circ}$ & $\begin{array}{l}\text { TEMPE } \\
\text { MEDI } \\
\text { MINIM }\end{array}$ & $\begin{array}{l}\text { ATURA } \\
\text { ANUAL } \\
\text { MAXTM }\end{array}$ & $\begin{array}{c}\text { PRECIPI- } \\
\text { TACION } \\
(\mathrm{mm})\end{array}$ & $\begin{array}{l}\text { LAT. } \\
\text { (L.S.) }\end{array}$ & $\begin{array}{l}\text { LONG. } \\
\text { (L.W.) }\end{array}$ & $\begin{array}{l}\text { ALT. } \\
\text { (msnm) }\end{array}$ \\
\hline 1 & W/mmera R/Elmhurst & VIC & 8 & 8 & 19 & 617 & $37 \times 13^{\prime}$ & $143^{\circ} 16^{\prime}$ & 325 \\
\hline 2 & Wimmera R/Glenorchi & VIC & 12 & 9 & 22 & 448 & $36^{\circ} 55^{\prime}$ & $142^{\circ} 34^{\prime \prime}$ & 170 \\
\hline 3 & Lake Hindmarsh & VIC & 10 & 8 & 22 & 419 & $36^{\circ} 08^{\prime}$ & $141^{\circ} 27$ & 75 \\
\hline 4 & Outlet Creek Rainbow & VIC & 13 & 8 & 22 & 419 & $35^{\circ} 49^{\prime}$ & $141^{\circ} 36$ & 75 \\
\hline 5 & S Lake Albacutya & VIC & 12 & 8 & 22 & 419 & $35^{\circ} 48^{\prime}$ & $141^{\circ} 3 \sigma^{\circ}$ & 70 \\
\hline 6 & E. Lake Albacutya & VIC & 11 & 8 & 22 & 419 & $35^{\circ} 46^{\circ}$ & $142^{\circ} 25^{\prime}$ & 70 \\
\hline 7 & N Lake Albacutya & VIC & 12 & 8 & 22 & 419 & $35^{\circ} 42^{\prime}$ & $141^{\circ} 21^{\prime}$ & 70 \\
\hline 8 & Lowan Valley-Saline & VIC & 10 & 9 & 22 & 448 & $35^{\circ} 50^{\circ}$ & $141^{\circ} 48^{\prime}$ & 135 \\
\hline 9 & Avon River-Navarre & VIC & 10 & 8 & 19 & 617 & $36^{\circ} 52$ & $143^{\circ} 57$ & 280 \\
\hline 10 & Umberumberka Creek & NSW & 10 & 12 & 27 & 254 & $31^{\circ} 53^{\prime}$ & $141^{\circ} 56^{\prime}$ & 210 \\
\hline 11 & Port Lincoln & SA & 10 & 12 & 21 & 486 & $34^{\circ} 35^{\prime}$ & $135^{\circ} 38^{\prime}$ & 90 \\
\hline 12 & Katherine & NT & 12 & 20 & 34 & 952 & $14^{\circ} 29$ & $132^{\circ} 15^{\prime}$ & 95 \\
\hline 13 & Katherine & NT & 8 & 20 & 34 & 952 & $14^{\circ} 26^{\prime}$ & $132^{\circ} 18^{\circ}$ & 95 \\
\hline 14 & Eccles Creek-Petford & QLD & 10 & 20 & 26 & 800 & 17017 & $145^{\circ} 03^{\prime}$ & 500 \\
\hline 15 & Pinnacle Creek-Pet & QLD & $s$ & 14 & 26 & 800 & 17013 & $145^{\circ} 01^{\prime}$ & 460 \\
\hline 16 & Mishap Creek-Petford & QLD & 5 & 14 & 26 & 800 & 17011 & $145^{\circ} 07$ & 520 \\
\hline 17 & Eureka Creek-Petford & QLD & 5 & 14 & 26 & 800 & $17^{\circ} 15^{\prime}$ & $145^{\circ} 05^{\circ}$ & 520 \\
\hline 18 & Wales Siding-Petford & QLD & 10 & 14 & 26 & 873 & $17^{\circ} 22^{\prime}$ & $145^{\circ} 12^{\prime}$ & 780 \\
\hline 19 & Emu Creek-Petford & QLD & 5 & 14 & 26 & 800 & $17{ }^{\circ} 1^{\prime}$ & $144^{\circ} 57$ & 460 \\
\hline 20 & Emu Gibbs-Petford & QL.D & 5 & 14 & 26 & 1.116 & $175^{\prime}$ & $145^{\circ} 02^{\prime}$ & 500 \\
\hline 21 & Gibbs-Petford & QLD & 5 & 14 & 26 & 873 & $175^{\prime}$ & $145^{\circ} 11^{\prime}$ & 700 \\
\hline 22 & Chinamans-Petford & QLD & 5 & 14 & 26 & 1.116 & $17 \times 24$ & $145^{\circ} 1 \sigma^{\circ}$ & 680 \\
\hline 24 & Emu Creek-Petford & QLD & 3 & 14 & & 873 & $17^{\circ} 26^{\prime}$ & $145^{\circ} 02^{\prime}$ & 520 \\
\hline
\end{tabular}

(1) VIC Victoria

NSW : New South Wales

NT : North Territory

SA : South Australia

QLD : Queensland.

$N^{\circ}$ Numero de progenies en la procedencia.

\section{Ensayos Considerados}

Los ensayos considerados en este estudio fueron establecidos en 1989 y 1991, en las cuatro localidades que se señalan en el Cuadro $\mathrm{N}^{\circ} 2$.

Los cuatro ensayos fueron establecidos con plantas producidas en macetas en el vivero del Instituto Forestal, sobre sitios planos a ligeramente ondulados. con una intensiva preparación de suelo. Ellos han sido manejados con un esquema de silvicultura intensiva que incluye control de malezas y fertilización. 


\section{Cuadro $\mathrm{N}^{\mathrm{2}} 2$.}

UBICACIÓN DE LOS ENSAYOS DE Eucalyptus camaldulensis ESTABLECIDOS EN CHILE.

\begin{tabular}{|l|c|c|c|c|}
\hline ENSAYO & MEL-MEL & LONGOTOMA & TANTEHUE & LA PAILA \\
& $(1)$ & $(2)$ & $(3)$ & $(4)$ \\
\hline Región & $\mathrm{V}$ & $\mathrm{V}$ & RM & VI \\
Provincia & Valparaiso & Petorca & Santiago & Colchagua \\
Comuna & Casablanca & La Ligua & Melipilla & La Paila \\
Latitud S. & $33^{\circ} 23^{\circ}$ & $32^{\circ} 21^{\circ}$ & $33^{\circ} 32^{\circ}$ & $34^{\circ} 43^{\prime}$ \\
Longitud W. & $71^{\circ} 24^{\circ}$ & $71^{\circ} 26^{\circ}$ & $71^{\circ} 14^{\circ}$ & $71^{\circ} 39^{\prime}$ \\
Altitud (msnm) & 250 & 58 & 240 & 300 \\
Año de Establec. & 1989 & 1989 & 1991 & 1991 \\
Prec. Med. Anual $(\mathrm{mm})$ & 455 & 308 & 419 & 398 \\
Temp. Med. Anual $\left({ }^{\circ} \mathrm{C}\right)$ & 13,3 & 14,3 & 13,6 & 14,1 \\
Periodo Seco $\left(\mathrm{N}^{\circ}\right.$ meses) & $7-9$ & $7-9$ & 8 & 7 \\
\hline
\end{tabular}

\section{Diseño Experimental.}

El diseño experimental empleado en todos estos ensayos es el de "Bloques de Familias Compactas", o de parcelas divididas, en donde la parcela principal es la procedencia. Las familias se distribuyen al azar en subparcelas de 4 plantas en línea, dentro de cada procedencia. El diseño de cada ensayo consta de 10 repeticiones. Los ensayos fueron rodeados por dos líneas de aislación.

\section{Análisis Estadístico.}

Para determinar las diferencias en el desarrollo de las plantas tanto a nivel de procedencias como de familias, se realizaron análisis de varianza. Las comparaciones entre medias se efectuaron empleando la Prueba de Scheffe. En algunos de los ensayos sólo se consideró parte de la información, por estimarse que la inclusión de algunos bloques distorsionaría los resultados.

Además se estimaron la heredabilidad $\left(h^{2}\right)$ y las correlaciones genćticas y fenotipicas entre las variables estudiadas: ALT. DAC para los ensayos 1 y 2, y ALT, DAP y $D^{2} \mathrm{H}$, para los ensayos 3 y 4.

El modelo estadístico utilizado en la estimación de los parámetros genéticos en cada uno de los ensayos fue un modelo anidado completamente al azar, considerando los siguientes factores (Becker. 1988):

$$
y_{i k j l}=\mu+b_{i}+p_{j}+f(p)_{j k}+b_{1} x p_{j}+b_{i} x f(p)_{j k}+E_{i j k l}
$$

donde: 


$\begin{array}{lll}\mu & = & \text { Media poblacional. } \\ y_{\mathrm{ikjl}} & = & \text { Variable en estudio. } \\ \mathrm{b}_{\mathrm{i}} & = & \text { Efecto aleatorio del i-ésimo bloque. } \\ \mathrm{p}_{\mathrm{j}} & = & \text { Efecto aleatorio de la j-ésima procedencia. } \\ \mathrm{f}(\mathrm{p})_{j \mathrm{k}} & = & \begin{array}{l}\text { Efecto aleatorio de la k-ésima familia dentro de la j-ésima } \\ \text { procedencia. }\end{array} \\ \mathrm{b}_{\mathrm{i}} \times \mathrm{p}_{\mathrm{j}} & = & \text { Efecto de la interacción entre bloque y procedencia. } \\ \mathrm{b}_{1} \times \mathrm{f}(\mathrm{p})_{\mathrm{jk}} & = & \begin{array}{l}\text { Efecto de la interacción entre bloque y familia dentro de } \\ \text { procedencia. }\end{array} \\ \mathrm{E}_{\mathrm{j} \mathrm{jk}} & = & \text { Error experimental. }\end{array}$

La heredabilidad individual fue estimada siguiendo el mismo procedimiento de Whitemann et al. (1992), donde:

$$
h^{2}=\frac{2.86^{*} \text { Varianza } \cdot f}{\text { Varianzas } \cdot\left(b+f+b^{*} f(p)+\text { Error }\right)}
$$

El factor 2.86 se basa en el supuesto que la tasa de cruzamiento exogámico es de aproximadamente un $80 \%$. lo cual se considera normal en el caso de familias de polinización abierta. especialmente cuando la semilla se ha colectado en bosques nativos, lo que determina un coeficiente de parentesco de 0,35 : cuyo valor recíproco se asemeja a 2,86 .

La heredabilidad y correlación genética (con sus respectivos errores estandar) promedio ponderado de todos los ensayos se calculó según Cunningham et al. (1977), en cambio la correlación fenotípica promedio se obtuvo del promedio aritmético simple.

\section{RESULTADOS Y DISCUSIÓN.}

\section{Crecimiento Juvenil.}

Se entregan. separados por ensayo. los resultados promedios de sobrevivencia (\%). ALT $(\mathrm{m})$. DAC $(\mathrm{cm})$ y $\mathrm{D}^{2} \mathrm{H}\left(\mathrm{m}^{3}\right)$, para cada una de las procedencias.

\section{- Ensayo 1 (Mel-Mel)}

Los resultados de este ensayo, cvaluado a los 44 meses de edad se presentan en cl Cuadro $\mathrm{N}^{\circ} 3$. Se puede observar que. el testigo presenta una sobrevivencia de sólo de 
$62,5 \%$. La altura promedio es similar a la del ensayo Longotoma (2), correspondiendo el mayor valor, al igual que en los dos ensayos restantes, a la procedencia S. Lake Albacutya. Por su parte, y manteniendo la tendencia de los otros ensayos, el testigo nacional presentó una de las alturas menores. superando sólo a la procedencia Umberumberka Creek. que es la que exhibe el menor crecimiento en el ensayo.

Cuadro $\mathrm{N}^{\circ} 3$.

SOBREVIVENCIA (\%), ALTIRA (m) Y DIAMETRO A LA ALTURA DEL CUELIO (cm) A LOS 44 MESES DE EDAD DE Eucalyptus camaldulensis, ENSAYO DE MEL-MEI, V REGION.

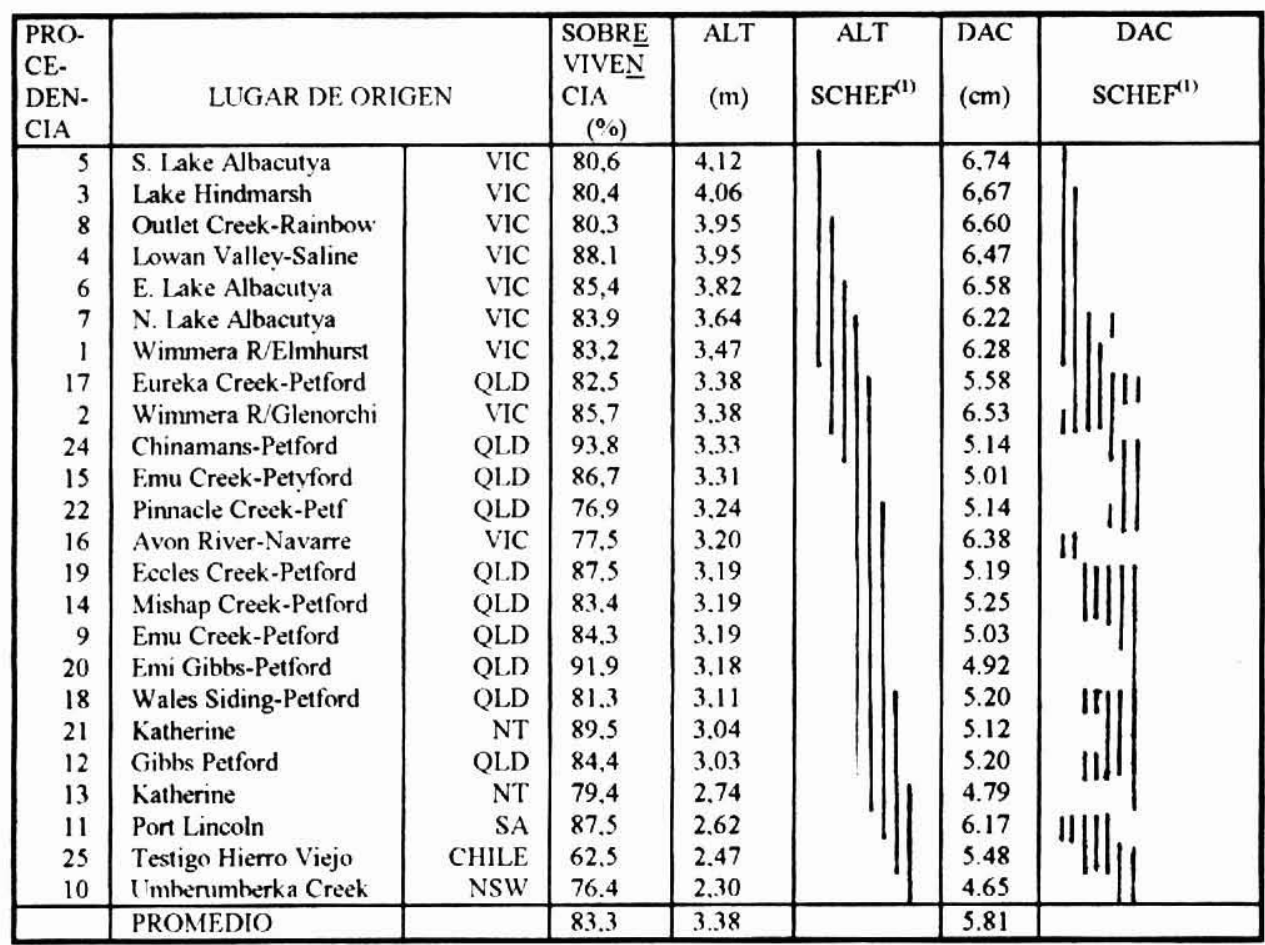

(1): Diferencias estadistica entre procedencias segun prueba de Scheffe( $p \leq 0.0001)$.

Los DAC promedios fluctuaron desde $6.74 \mathrm{~cm}$ (S. Lake Albacutya-VIC) a sólo 4,65 $\mathrm{cm}$ (Umberumberka Creek-NSW). equivalente a un $45 \%$ de diferencia entre ambas procedencias. Las diferencias significativas entre procedencias indican que las mejores fueron todas originarias de VIC. Umberumberka Creek fue la de menor DAC en este ensayo. 
En el análisis familiar se determinaron las mejores 30 familias según $D^{2} H$, corrigiendo esta información por bloque y procedencia (Cuadro $\mathrm{N}^{\circ} 4$ ). La familia que presentó significativamente mejores crecimientos proviene de Queensland y corresponde a Wales Siding-Petford (167), seguida de la familia 50 del Sur de Lake Albacutya (VIC). Este mayor crecimiento esta determinado principalmente por la altura. ya que los diámetros de las mejores familias fueron semejantes.

\section{Cuadro $\mathrm{N}^{\circ} 4$.}

ORDENAMIENTO DE LAS 30 MEJORES FAMILIAS SEGÚN D² H AJUSTADO POR BLOQUE Y PROCEDENCIA PARA EL ENSAYO DE MEL-MEL.

\begin{tabular}{|c|c|c|c|c|c|c|}
\hline ORD & PROCEDENCIA & UBIC. & $\begin{array}{c}\text { FAMIL. } \\
\mathrm{N}^{\circ}\end{array}$ & $\begin{array}{c}\text { ALTURA } \\
(\mathrm{m})\end{array}$ & $\begin{array}{l}\text { DAC } \\
(\mathrm{cm})\end{array}$ & $\begin{array}{l}\mathrm{D}^{2} \mathrm{H} \\
\left(\mathrm{m}^{3}\right)\end{array}$ \\
\hline 1 & Wales Siding-Petford & QLD & 167 & 4,19 & 6.68 & 0.0210 \\
\hline 2 & S. L ake Albacutya & VIC & 50 & 3,71 & 6.61 & 0,0189 \\
\hline 3 & Lake Hindmarsh & VIC & 26 & 3,72 & 6,33 & 0.0187 \\
\hline 4 & Port Lincoln & SA & 112 & 3,91 & 6.56 & 0.0179 \\
\hline 5 & Mishap Creek-Petford & QLD & 156 & 3,62 & 6,33 & 0.0177 \\
\hline 6 & Avon River-Navarre & VIC & 91 & 3,40 & 6.46 & 0,0166 \\
\hline 7 & E. Lake Albacutya & VIC & 60 & 3.79 & 6.05 & 0,0166 \\
\hline 8 & Lowan Valley-Saline & VIC & 81 & 3,94 & 5.99 & 0.0164 \\
\hline 9 & Outlet Creek Rainbow & VIC & 33 & 3.67 & 6,16 & 0,0160 \\
\hline 10 & S. Lake Albacutya & VIC & 46 & 3,92 & 6,08 & 0,0159 \\
\hline 11 & Outlet Valley-Saline & vic & 36 & 3,69 & 5,78 & 0,0158 \\
\hline 12 & Lake Hindmarsh & VIC & 29 & 3,12 & 5,48 & 0.0158 \\
\hline 13 & S. Lake Albacutya & VIC & 51 & 3,56 & 6,20 & 0,0156 \\
\hline 14 & N. Lake Albacutya & VIC & 67 & 3,60 & 6,28 & 0.0155 \\
\hline 15 & N. Lake Albacutya & VIC & 68 & 3.48 & 6,15 & 0.0155 \\
\hline 16 & Wimmera R/Elmhurst & VIC & 3 & 3,47 & 6,25 & 0,0154 \\
\hline 17 & Wales Siding-Petford & QLD & 168 & 3.65 & 6,36 & 0,0154 \\
\hline 18 & Umberumberka Creek & NSW & 101 & 3.29 & 6,42 & 0.0150 \\
\hline 19 & E. Lake Albacutya & VIC & 58 & 3,41 & 6,10 & 0.0148 \\
\hline 20 & E. I ake Albacutya & VIC & 62 & 3,54 & 6.00 & 0.0147 \\
\hline 21 & Katherine & NT & 124 & 3.59 & 6,10 & 0,0147 \\
\hline 22 & Mishap Creek-Petford & QLD & 154 & 3,45 & 5.64 & 0.0146 \\
\hline 23 & E. Lake Albacutya & VIC & 61 & 3,61 & 5,93 & 0,0145 \\
\hline 24 & Wimmera R/Glenorchi & VIC & 17 & 3,08 & 6.26 & 0,0145 \\
\hline 25 & Emu Creek Petford & QLD & 178 & 3,61 & 6,09 & 0,0144 \\
\hline 26 & Avon River-Navarre & VIC & 96 & 3,49 & 5,88 & 0,0143 \\
\hline 27 & Katherine & NT & 132 & 3.47 & 6.04 & 0,0142 \\
\hline 28 & Avon River-Navarre & VIC & 95 & 3,25 & 6.20 & 0,0141 \\
\hline 29 & Lake Hindmarsh & VIC & 24 & 3,30 & 5,82 & 0,0140 \\
\hline \multirow[t]{2}{*}{30} & Emu Gibbs-Petford & QLD & 181 & 3.39 & 6.00 & 0,0139 \\
\hline & PROMEDIO & & & 3.58 & 6.17 & 0.0157 \\
\hline
\end{tabular}

Estas mejores 30 familias son superiores en promedio de altura y diámetro en 5.9 y 19,1\%. respectivamente, al promedio del ensayo. Por otra parte, entre las 30 familias superiores estan representadas procedencias de todos los origenes, VIC, QLD, NT y NSW. siendo las familias provenientes de Lake Albacutya las más frecuentes. 
La diferencia que existe entre las 10 familias de mejor y peor crecimiento se entrega en la Figura $\mathrm{N}^{\circ} 1$, siendo significativas las diferencias entre familias para el $\mathrm{D}^{2} \mathrm{H}$ ( $\mathrm{p} \leq$ 0,0003 ). La superioridad de la familia de mayor crecimiento (167) respecto a la familia de menor crecimiento (111) es de un $\mathbf{2 8 6 , 8 \%}$. Importante es destacar que familias procedentes de Lake Albacuyta presentan muy buenos $(34,60,46)$ y también muy malos crecimientos $(49.77,57)$.

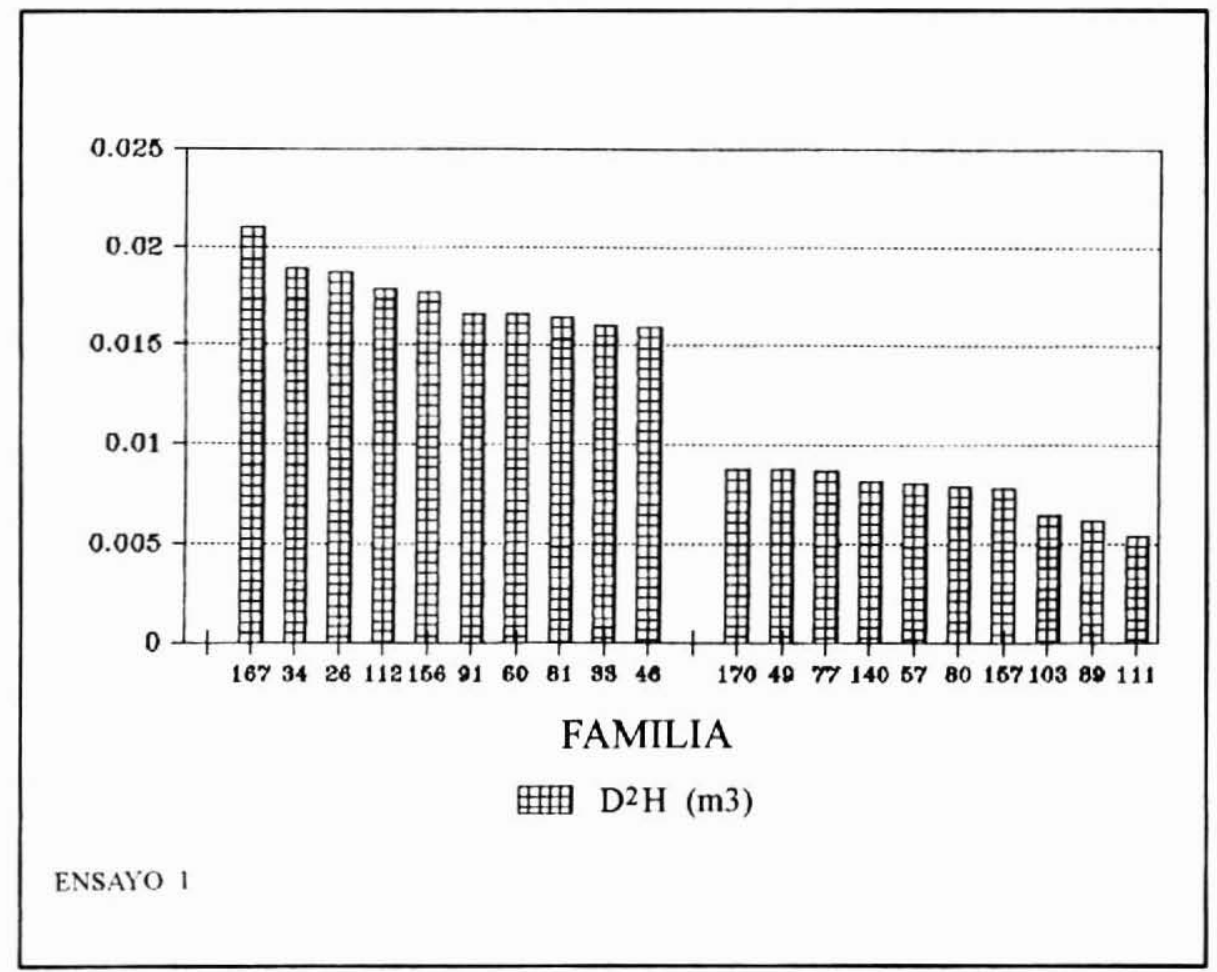

Figura $\mathrm{N}^{\circ}$ 1. E. Calmaldulensis - MEL-MEI. MFJORES Y PEORES 10 FAMILIAS SEGÚN $\mathrm{D}^{2} \mathrm{H}$

- Ensayo 2 (Longotoma)

Después de 43 meses, los resultados del ensayo Longotoma (V Región) indican que la ALT promedio fue de $3.28 \pm 1,11 \mathrm{~m}$. el DAC $5.24 \pm 2,00 \mathrm{~cm}$ y la SOB de $93,2 \%$ (Cuadro $\mathrm{N}^{\circ} 5$ ), determinándose diferencias significativas entre las procedencias para ALT y DAC $(\mathrm{p} \leq 0.0001)$. Entre las procedencias también se evaluó el comportamiento de un origen nacional (Hierro Viejo, V Región). 
La sobrevivencia obtenida en Longotoma por las procedencias australianas fluctuó entre $97.8 \%$ (Lowan Vallcy-Saline. VIC) y $85,8 \%$ (Emu Creek-Petford, QLD). Sin embargo. la procedencia nacional sólo alcanzó un $65 \%$. siendo levemente superior a la alcanzada en el ensayo de Mel-Mel (1).

\section{Cuadro $\mathrm{N}^{\circ} 5$.}

SOBREVIVENCIA, ALTURA (ALT) Y DIȦMETRO A LA ALTI'RA DEL CUELLO (DAC) A LOS 44 MESFS DE EDAD DE Eucalyptus camaldulensis, ENSAYO DE LONGOTOMA, V REGIÓN (ORDENADAS SEGUUN ALTURA).

\begin{tabular}{|c|c|c|c|c|c|c|c|}
\hline PROCED & \multicolumn{2}{|c|}{ LUGAR DE ORIGEN } & $\begin{array}{l}\text { SOBRE- } \\
\text { VIV } \\
(\%)\end{array}$ & $\begin{array}{l}\text { AL.T } \\
\text { (m) }\end{array}$ & $\begin{array}{c}\text { ALT } \\
\text { SCHEF(1) }\end{array}$ & $\begin{array}{l}\text { DAC } \\
(\mathrm{cm})\end{array}$ & $\begin{array}{c}\text { DAC } \\
\text { SCHFF' }\end{array}$ \\
\hline 5 & S Lake Albacutya & VIC & $\$ 6.8$ & 4,05 & & 0,19 & \\
\hline 3 & Lake Hindmarsh & VIC & 93,1 & 3,85 & I & 6.04 & \\
\hline 4 & Outlet Crekk Rainbow & VIC & क.5 & 3,61 & 1 & 5.46 & \\
\hline 19 & Emu Creek-Petford & QLD & $\$ 1.0$ & 3.51 & & 5,45 & \\
\hline 6 & E Lake Albacutya & VIC & 93.0 & 3,44 & 1 & 5,53 & \\
\hline 21 & Gibbs-Petford & QLD & 03,5 & 3,39 & & 5,34 & 1 \\
\hline 20 & Emu Gibbs-Petford & QLD & 05,6 & 3,38 & & 4.95 & \\
\hline 18 & Wales Siding-Petford & QLn & 05,4 & 3,37 & 1 & 5.18 & 11 \\
\hline 8 & N Lake Alhacurya & VIC & 80.0 & 3.36 & & 5.58 & \\
\hline 7 & Lowan Valley-Saline & VIC & 07.8 & 3.35 & & 5,40 & \\
\hline 24 & Emu Creek-Petford & QL.D & 85.8 & 3.28 & & 4,79 & 1 \\
\hline 17 & Eureka Creek-Petford & QI.D & 035 & 3.27 & 1 & 4.76 & \\
\hline 22 & Chunamans-Petford & QLD & 88.5 & 3.27 & & 4.75 & \\
\hline 12 & Kathenne & NT & $04, R$ & 3.25 & & 5,12 & II \\
\hline 14 & Eccles Creek-Petford & QLD & 01.1 & 3.23 & & 4,59 & \\
\hline 15 & Pinnacle Crekk-Petf & QI.D & 07.0 & 3.21 & & 4.70 & 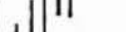 \\
\hline 1 & Wimmera R/Elmhrust & VIC & 02,5 & 3.10 & & 5.57 & \\
\hline 2 & Wimmera R/Glencrch & QI.D & 94.8 & $3.0 R$ & & 5.67 & 111 \\
\hline 10 & Mishap Creek-Petford & QLD & 80.4 & 2,96 & -1 & 4,05 & \\
\hline 13 & Kathenner & NT & 92,5 & 2.80 & & 4.01 & \\
\hline 9 & Avon River-Navarte & VIC & 88.1 & 2.70 & & 5.11 & 11 \\
\hline 25 & Hierro Viejo V Rer & CHII.E. & 05.0 & 2.01 & 1 & 4,63 & 101 \\
\hline 11 & Port Lincoln & SA & 02,5 & 2.41 & 1 & 5,34 & $\| 11$ \\
\hline \multirow[t]{2}{*}{10} & Umbenumberka Creek & WNS & 94,4 & 2.34 & & 4,07 & 1 \\
\hline & PROMEDIO & & 0.3 .2 & 3.28 & & 5.24 & \\
\hline
\end{tabular}

(1) : Diferencias estadisticamente significativas seguin prueba deScheffe ( $p \leq 0.0001)$.

Una ordenación descendente de las 30 mejores familias según su valor para la variable $D^{2} \mathrm{H}$ (Cuadro $\mathrm{N}^{\circ} 6$ ) deja en evidencia la gran representación en este grupo de las progenies provenientes del estado de Victoria. Por otra parte. y como guia para la selección. estas familias representan en promedio un $6.4 \%$ y $10,31 \%$ de incremento en altura y diámetro. respectivamentc. en relación a los valores promedios del ensayo completo.

La variación en crecimiento entre las progenies es considerable. como se puede apreciar en la Figura $\mathrm{N}^{\circ} 2$. De igual forma. la variación entre progenies dentro de las procedencias también es importante. existiendo procedencias como Avon River Navarre (VIC) que presenta progenies ubicadas entre las de mejor crecimiento (familia 97) y a su vez a la familia de menor crecimiento en todo el ensayo (familia 89). 
Cuadro $\mathrm{N}^{\circ} 6$.

ORDENAMIENTO DE LAS 30 MEJORES FAMILIAS SEGÚN DH AJUSTADO POR BLOQUE Y PROCEDENCIA PARA EL ENSAYO DE LONGOTOMA.

\begin{tabular}{|c|c|c|c|c|c|c|}
\hline ORD & PROCEDENCIA & UBIC. & $\begin{array}{c}\text { FAMIL- } \\
\mathrm{N}^{\circ}\end{array}$ & $\begin{array}{l}\text { ALT } \\
(\mathrm{m})\end{array}$ & $\begin{array}{l}\text { DAC } \\
(\mathrm{cm})\end{array}$ & $\begin{array}{l}D^{2} \mathrm{H} \\
\left(\mathrm{m}^{3}\right)\end{array}$ \\
\hline 1 & Outlet Creek-Rainbow & VIC & 33 & 3,75 & 6.29 & 0.0229 \\
\hline 2 & Wimmera R/Elmhurst & VIC & 3 & 3,57 & 6,71 & 0,0193 \\
\hline 3 & Lowan Valley-Saline & VIC & 79 & 3,61 & 6,18 & 0,0186 \\
\hline 4 & Wimmera R/Elmhurst & VIC & 5 & 3,65 & 6,55 & 0,0178 \\
\hline 5 & Avon River-Navarre & VIC & 97 & 3,50 & 5,95 & 0,0173 \\
\hline 6 & Lake Hindmarsh & VIC & 30 & 3,47 & 5,35 & 0.0169 \\
\hline 7 & E. Lake Albacutya & VIC & 62 & 3.81 & 5,97 & 0,0168 \\
\hline 8 & Lowan Valley-Saline & VIC & 86 & 3,44 & 5,76 & 0.0167 \\
\hline 9 & E. Lake Albacutya & VIC & 59 & 3,78 & 6.00 & 0.0165 \\
\hline 10 & Wimmera R/Elmhurst & VIC & 2 & 3.30 & 6,31 & 0,0164 \\
\hline 11 & Wimmera R/Elmhurst & VIC & 4 & 3,21 & 6,54 & 0,0162 \\
\hline 12 & Emu Creek-Petford & QLD & 177 & 3,26 & 4,48 & 0.0156 \\
\hline 13 & Avon River-Navarre & VIC & 95 & 3,61 & 5,53 & 0,0155 \\
\hline 14 & Lake Hindmarsh & VIC & 26 & 3,29 & 5,60 & $0,0] 53$ \\
\hline 15 & Pinnacle Creek-Petf & QLD & 152 & 3,46 & 5,54 & 0,0152 \\
\hline 16 & Eccles Creek-Petford & QI.D & 143 & 3,42 & 5.77 & 0.0151 \\
\hline 17 & S. Lake Albacutya & VIC & 45 & 3,47 & 5,78 & 0,0150 \\
\hline 18 & Lake Hindamrsh & VIC & 25 & 3,43 & 6.00 & 0,0150 \\
\hline 19 & N. L ake Albacutya & VIC & 73 & 3,56 & 5,78 & 0,0145 \\
\hline 20 & Wimmera R/Glenorchi & VIC & 16 & 3,73 & 5,79 & 0,0145 \\
\hline 21 & Wimmera R/Glenorchi & VIC & 14 & 3.46 & 5,70 & 0,0145 \\
\hline 22 & Lowan Valley-Saline & VIC & 81 & 3,41 & 5,83 & 0.0144 \\
\hline 23 & Avon River-Navarre & VIC & 91 & 3.70 & 5,46 & 0,0143 \\
\hline 24 & S. Lake Albacutya & VIC & 51 & 3,28 & 5,58 & 0.0142 \\
\hline 25 & Outlet Creek-Rainhow & VIC & 38 & 3,40 & 5,52 & 0.0142 \\
\hline 26 & Katherine & $\mathrm{NT}$ & 124 & 3,41 & 5.22 & 0.0139 \\
\hline 27 & E. Lake Albacutya & VIC & 65 & 3,37 & 5,74 & 0.0138 \\
\hline 28 & Chinamans-Petford & QL.D & 192 & 3,46 & 5.26 & 0,0138 \\
\hline 29 & Wimmera R/Glenorchi & VIC & 10 & 3.41 & 5.37 & 0.0138 \\
\hline \multirow[t]{2}{*}{30} & Lake Hindmarsh & VIC & 27 & 3,54 & 5.75 & 0.0138 \\
\hline & PROMEDIO & & & 3.49 & 5.78 & 0.0157 \\
\hline
\end{tabular}




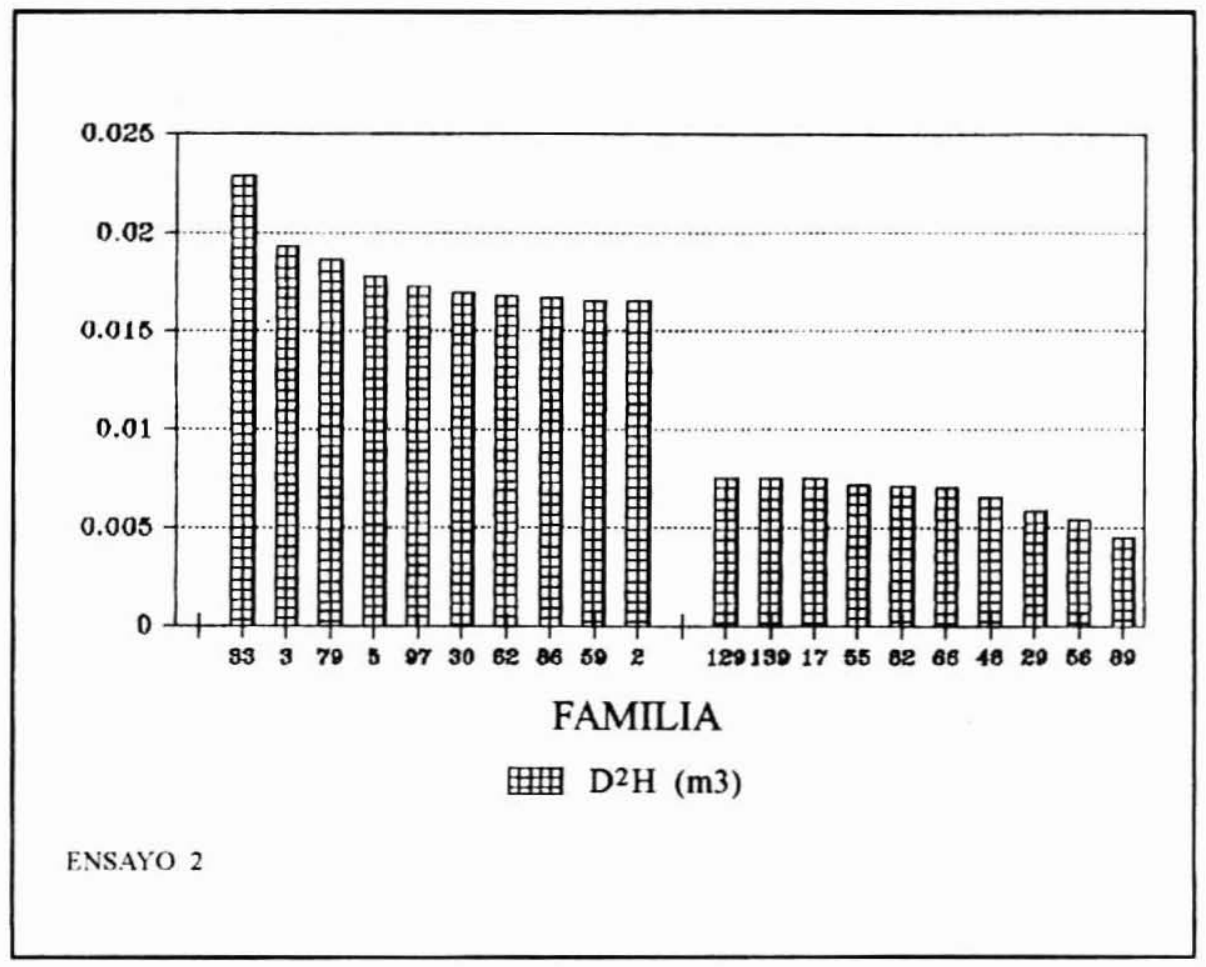

Figura $N^{\circ}$ 2. E. Calmaldulensis - LONGOTOMA MEJORES Y PEORES 10 FAMILIAS SEGÚN D²H

- Ensayo 3 (Tantehue)

En este ensayo se compararon sólo procedencias de Victoria con un testigo nacional, a base de la información de una evaluación a los $\mathbf{4 2}$ meses de edad. Los valores medios registrados para las variables en estudios (Cuadro $\mathrm{N}^{\circ} 7$ ) son: Altura $6,59 \pm 1,76 \mathrm{~m}$; DAP $6,41 \pm 2,0 \mathrm{~cm} ; \mathrm{D}^{2} \mathrm{H}$ de $0.033 \pm 0.03 \mathrm{~m}^{3}$ y SOB de $96.9 \%$. Estos valores, que fueron obtenidos considerando todos los bloques, son los mayores de entre los cuatro ensayos considerados en el análisis. lo que obedece a las mejores condiciones de sitio imperantes en el lugar y a que el año de establecimiento fue benigno en materia de lluvias, situación que no se observó en los ensayos de Mel-Mel (1) y Longotoma (2) que debieron soportar un año de sequia. 


\section{Cuadro $N^{0} 7$.}

SOBREVIVENCLA (\%), ALTURA (m) Y DIAMETRO A LA ALTURA DEL PECHO (cm) A LOS 42 MESES DE EDAD DE Eucalyptus camaldulensis, ENSAYO DE TANTEHUE, V REGION (ordenados seguin $D^{2} H$ ).

\begin{tabular}{|c|c|c|c|c|c|c|c|}
\hline $\begin{array}{l}\text { PRO- } \\
\text { DEN- } \\
\text { CIA }\end{array}$ & \multicolumn{2}{|l|}{ LUGAR DE ORIGEN } & $\begin{array}{c}\text { SOBRE } \\
\text { VIVEN- } \\
\text { CIA } \\
(\%)\end{array}$ & $\begin{array}{l}\text { ALT } \\
\text { (m) }\end{array}$ & $\begin{array}{l}\text { DAP } \\
(\mathrm{cm})\end{array}$ & $\begin{array}{l}D^{2} \mathrm{H} \\
\left(\mathrm{m}^{3}\right)\end{array}$ & $\begin{array}{c}\mathrm{D}^{2} \mathrm{H} \\
\text { SCHEF }^{(1)}\end{array}$ \\
\hline 5 & S. Lake Albacutya & VIC & 98,6 & 7.12 & 6.79 & 0,0401 & \\
\hline 3 & Lake Hindmarsh & VIC & 96,5 & 6.81 & 6.76 & 0,0390 & \\
\hline 8 & Lowan Valley-Saline & VIC & 91,9 & 6.72 & 6.72 & 0,0386 & \\
\hline 6 & E. Lake Albacutya & VIC & 95.3 & 6.70 & 6.74 & 0,0373 & \\
\hline 7 & N. Lake Albacutya & VIC & 97,7 & 6.82 & 6.64 & 0,0356 & \\
\hline 1 & Wimmera R/Elmhurst & VIC & 96.5 & 6.38 & 6.26 & 0.0322 & \\
\hline 4 & Outlet Creek-Rainbow & VIC & 99.0 & 6.60 & 6.07 & 0,0286 & \\
\hline 2 & Wimmera R/Glenorchi & VIC & 95,0 & 6.25 & 6,10 & 0,0282 & \\
\hline 9 & Avon River-Navarre & VIC & 95.8 & 6,06 & 6,10 & 0,0281 & \\
\hline 25 & Textigo Lolol & CHILE & 100.0 & 5.36 & 4.66 & 0.0148 & I \\
\hline & PROMEDIO & & 96.9 & 6.59 & 6.41 & 0.0334 & \\
\hline
\end{tabular}

11 : Diferencias estadisticamente significativas segun prueba deScheffe ( $p \leq 0,0001)$.

La mayor sobrevivencia la expresa el testigo nacional (100\%), pero no se diferencia mayormente de la SOB promedio del ensayo. la que es en general alta $(96,9 \%)$. La menor SOB corresponde a la procedencia Lowan Valley Saline $(91.9 \%)$

La altura promedio del ensayo alcanzó los 6.59 metros, variando entre 7,12 m (S. Lake Albacutya) y $5.36 \mathrm{~m}$ (testigo nacional). Se manifiesta una clara inferioridad de las plantas de semilla local, las cuales exhiben entre un 24.7 y $11.5 \%$ menos de altura que la mejor y peor procedencia australiana. respectivamente.

Las procedencias mostraron diferencias significativas también en el DAP ( $\mathrm{p} \leq$ 0.0001 ), las cuales variaron desde $6.79 \mathrm{a} 4.66 \mathrm{~cm}$. para las procedencias originarias de VIC: S. Lake Albacutya y Avon River-Navarre, respectivamente (Cuadro $\mathrm{N}^{\circ} 7$ ). En gencral las procedencias de mayor DAP fueron las de mayores alturas. Nuevamente el testigo nacional demuestra el menor DAP $(4.66 \mathrm{~cm})$. nivel bastante inferior al promedio del ensayo.

Finalmente en el ensayo de Tantehue (3). las procedencias con mayores $D^{2} \mathrm{H}$ promedios son: Sur Lake Albacutya, Lake Hindmarsh y de N. Lake Albacutya, siendo la testigo nacional muy diferente al resto de las procedencias. El $D^{2} \mathrm{H}$ de la procedencia nacional muestra el menor crecimiento $y$ difiere significativamente de todas las procedencias australianas ( $\mathrm{p} \leq 0,0001$ ). siendo incluso un $36.8 \%$ inferior a la mejor procedencia del Sur de Lake Albacutya (Victoria). 
El ordenamiento de las mejores 30 familias por $D^{2} \mathrm{H}$, corregidos los efectos significativos de bloque y procedencia, según los promedios de mínimos cuadrados por familia, se entregan en el Cuadro 8. Se encontraron diferencias significativas entre familias ( $p \leq 0,0001$ ) para las tres características analizadas (ALT, DAP y $D^{2} H$ ), situación muy importante de considerar al momento de realizar selección familiar, destacando la mayor importancia de obtener las mejores familias mediante un indice de selección que incluya todas estas características.

La superioridad promedio de estas 30 mejores familias corresponde a un $9,2 \%$ comparado con el promedio de todo el ensayo $\left(0,0031 \mathrm{~m}^{3}\right)$, diferencia importante para estimar el progreso genético a conseguir por selección familiar (Cuadro $\mathrm{N}^{\circ} 8$ ). La familia nacional alcanza un $\mathrm{D}^{2} \mathrm{H}$ de $0,029 \mathrm{~m}^{3}$ ocupando el lugar 46 de las 83 familias evaluadas en este ensayo. es decir, es inferior en $39.7 \%$ comparado con la mejor familia (E. Lake Albacutya).

El rango que existe entre las 10 familias de mejor y peor crecimiento se entrega enla Figura $\mathrm{N}^{0} 3$. Estas diferencias significativas entre familias $(\mathrm{p} \leq 0.0001)$ fluctuan entre 0,048 y $0,011 \mathrm{~m}^{3}$. lo que significa una superioridad sobre el $336 \%$ de la mejor familia a la de menor crecimiento. Nuevamente. se observa una amplia variabilidad de las familias dentro de las procedencias. existiendo algunas procedencias que cuentan con familias tanto en el grupo de las 10 con mejor $D^{2} \mathrm{H}$. como en el grupo de los $10 \mathrm{D}^{2} \mathrm{H}$ más bajos. 


\section{Cuadro $\mathrm{N}^{\circ} 8$.}

ORDENAMIENTO DE LAS 30 MEJORES FAMILIAS SEGÚN DH AJUSTADO POR BLOQUE Y PROCEDENCIA PARA EL ENSAYO DE TANTEHUE.

\begin{tabular}{|c|c|c|c|c|c|c|}
\hline ORD & PROCEDENCIA & UBIC. & $\begin{array}{c}\text { FAMIL. } \\
\mathrm{N}^{\circ}\end{array}$ & $\begin{array}{l}\text { ALT } \\
(\mathrm{m})\end{array}$ & $\begin{array}{l}\text { DAP } \\
(\mathrm{cm})\end{array}$ & $\begin{array}{l}D^{2} \mathrm{H} \\
\left(\mathrm{m}^{3}\right)\end{array}$ \\
\hline 1 & E. Lake Albacutya & VIC & 59 & 7,00 & 7,90 & 0,0480 \\
\hline 2 & Wimmera R/Elmhurst & VIC & 3 & 7,25 & 7,36 & 0,0449 \\
\hline 3 & Lake Hindmarsh & VIC & 22 & 7,08 & 7,32 & 0,0429 \\
\hline 4 & N. Lake Albacutya & VIC & 67 & 7,16 & 7,22 & 0,0403 \\
\hline 5 & S. Lake Albacutya & VIC & 46 & 7,17 & 6,98 & 0,0403 \\
\hline 6 & Outlet Creek Rainbow & VIC & 33 & 6,60 & 7,19 & 0,0383 \\
\hline 7 & Wimmera R/Glenorchi & VIC & 13 & 7,08 & 6,80 & 0,0380 \\
\hline 8 & Avon River-Navarre & VIC & 95 & 6,90 & 6,98 & 0,0377 \\
\hline 9 & Avon River-Navarre & VIC & 92 & 7,19 & 6,85 & 0,0376 \\
\hline 10 & N. Lake Albacutya & VIC & 69 & 7,02 & 7,03 & 0,0374 \\
\hline 11 & Lowan Valley-Saline & VIC & 87 & 6,85 & 6,53 & 0,0372 \\
\hline 12 & S. Lake Albacutya & VIC & 44 & 7,08 & 6,84 & 0,0371 \\
\hline 13 & S. Lake Albacutya & VIC & 45 & 6,87 & 7,07 & 0,0370 \\
\hline 14 & Outlet Creek Rainbow & VIC & 42 & 7,00 & 6,88 & 0,0362 \\
\hline 15 & Lowan Valley-Saline & VIC & 85 & 6,95 & 6,56 & 0,0354 \\
\hline 16 & Lake Hindmarsh & VIC & 26 & 7,09 & 6,68 & 0,0350 \\
\hline 17 & Wimmera R/Glenorchi & VIC & 12 & 6.94 & 6,66 & 0,0349 \\
\hline 18 & Avon River-Navarre & VIC & 93 & 6,90 & 6,64 & 0,0348 \\
\hline 19 & Lowan Valley-Saline & VIC & 88 & 6,59 & 6,69 & 0,0346 \\
\hline 20 & N. Lake Albacutya & VIC & 73 & 6,89 & 6,58 & 0,0346 \\
\hline 21 & Wimmera R/Elmhurst & VIC & 4 & 7,02 & 6,57 & 0,0341 \\
\hline 22 & E. Lake Albacutya & VIC & 65 & 6.95 & 6,77 & 0,0340 \\
\hline 23 & N. Lake Albacutya & VIC & 70 & 7,04 & 6,57 & 0,0338 \\
\hline 24 & Avon River-Navarre & VIC & 97 & 6,55 & 6,68 & 0,0333 \\
\hline 25 & Wimmera R/Glenorchi & VIC & 19 & 6.49 & 6,73 & 0,0332 \\
\hline 26 & Wimmera R/Glenorchi & VIC & 15 & 6,60 & 6,62 & 0,0331 \\
\hline 27 & Lake Hindmarsh & VIC & 23 & 6,73 & 6,42 & 0,0331 \\
\hline 28 & S. Lake Albacutya & VIC & 53 & 6.38 & 6,86 & 0.0329 \\
\hline 29 & Lowan Valley-Saline & VIC & 81 & 7,12 & 6,35 & 0,0328 \\
\hline 30 & E. Lake Alhacutya & VIC & 60 & 7,14 & 6.35 & 0,0327 \\
\hline & PROMEDIO & & & 6.92 & 6.82 & 0.0365 \\
\hline
\end{tabular}




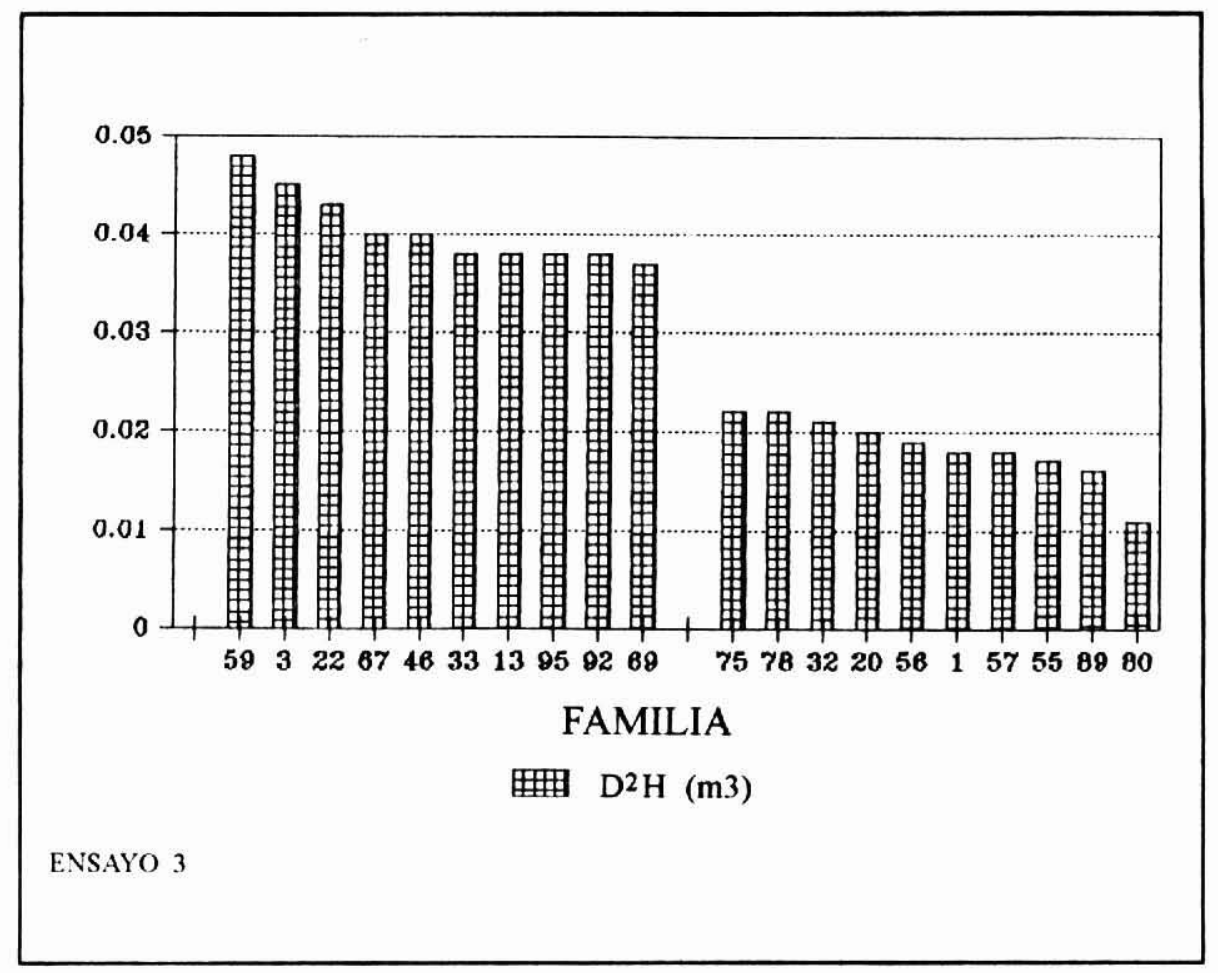

Figura $N^{\circ}$ 3. E. Calmaldulensis - TANTEHUE MEJORES Y PEORES 10 FAMILIAS SEGÚN D ${ }^{2} \mathrm{H}$

- Ensayo 4 (La Paila)

$\mathrm{Al}$ igual que el de Tantehue. este ensayo considera sólo procedencias y progenies provenientes del estado de Victoria y la información que se entrega corresponde a una medición realizada a los 42 meses de edad.. Los valores promedios y sus respectivas desviaciones para las variables en estudio fueron: $A L T=2,57 \pm 0,73 \mathrm{~m}$; $\mathrm{DAP}=2,48 \pm$ $1.07 \mathrm{~cm} . \mathrm{D}^{2} \mathrm{H}=0.0022 \pm 0.0027 \mathrm{~m}^{3}$ y SOB igual a 78,1\% (Cuadro $\mathrm{N}^{\circ}$ 9).

La sobrevivencia y desarrollo observados en este ensayo son los menores de los cuatro considerados en el análisis. Este comportamiento se puede explicar fundamentalmente por variación atribuible al ambiente, pues las familias evaluadas son casi las mismas contempladas en el ensayo Tantehue, sitio más favorable donde exhiben un desarrollo notablemente superior. 
Se observa que el testigo nacional presenta una de las menores sobrevivencias, superando sólo a una procedencia australiana (Avon River Navarre), y acusando nuevamente el menor crecimiento para el testigo.

Se determinó la existencia de diferencias estadísticamente significativas en las variables altura y diámetro entre las procedencias ensayadas ( $\leq \leq 0,0001)$. En el caso de la altura la diferencia entre los promedios de la mejor y peor procedencia (Lake Hindmarsh y testigo nacional, respectivamente) equivale a un $39,1 \%$. En el caso del diámetro, esta diferencia equivale al $55,5 \%$.

\section{Cuadro $N^{\circ} 9$.}

SOBREVIVENCIA (\%), ALTURA (m) Y DIAMETRO A LA ALTURA DEL PECHO (cm) A LOS 42 MFSES DE EDAD DE, Eucalyptus camaldulensis, ENSAYO DE LA PAILA, VI REGION (ordenados según $D^{2} H$ ).

\begin{tabular}{|c|c|c|c|c|c|c|c|}
\hline PRO & LUGAR DE ORIGEN & & $\begin{array}{c}\text { SOBR. } \\
(\%)\end{array}$ & $\begin{array}{l}\text { Al.T } \\
\text { (m) }\end{array}$ & $\begin{array}{l}\text { DAP } \\
(\mathrm{cm})\end{array}$ & $\begin{array}{l}\mathrm{D}^{2} \mathrm{H} \\
\left(\mathrm{m}^{3}\right)\end{array}$ & $\begin{array}{c}D^{2} H \\
\text { SCHEF }^{(1)}\end{array}$ \\
\hline 6 & E. Lake Albacutya & VIC & 70,4 & 2.58 & 2.60 & 0,002797 & \\
\hline 3 & Lake Hindmarsh & VIC & 79.5 & 2.74 & 2.66 & 0,002760 & \\
\hline 7 & N. Lake Albacutya & VIC & 82.0 & 2.67 & 2.66 & 0,002552 & \\
\hline I & Wimmera R/Elmhurst & VIC & 76.1 & 2.57 & 2.58 & 0,002409 & \\
\hline 8 & Lowan Valley-Saline & VIC & 73,8 & 2.69 & 2.58 & 0,002311 & \\
\hline 5 & S. Lake Albacutya & VIC & 76,9 & 2.72 & 2.54 & 0,002243 & \\
\hline 4 & Outlet Creek-Rainbow & VIC & 76.1 & 2.48 & 2.26 & 0,001810 & \\
\hline 2 & Wimmera R/Glenorchi & VIC & 76,7 & 2.33 & 2.28 & 0,001710 & \\
\hline 9 & Avon River-Navarre & VIC & 66.0 & 2.24 & 2.21 & 0.001322 & \\
\hline 27 & Testigo Lolol & CHILE & 70.0 & 1.97 & 1.71 & 0,000687 & \\
\hline & PROMEDIO & & 78.1 & 2.57 & 2.48 & 0.002235 & \\
\hline
\end{tabular}

(1): Diferencias estadistica entre procedencias segun prueba de Scheffe(p $\leq 0.0001)$

El comportamiento familiar en el ensayo La Paila se resume en el Cuadro $N^{\circ} 10$, donde se ordenaron las mejores 30 familias según $\mathrm{D}^{2} \mathrm{H}$, corregido por bloque $y$ procedencia. Es importante destacar que las mejores familias procedentes de VIC son: Lake Albacutya y Lake Hindmarsh. En general estas 30 familias son un 4,3; 7,5 y $15.7 \%$ superiores al promedio del ensayo en ALT. DAP y $D^{2} \mathrm{H}$, respectivamente. Se determinaron diferencias significativas entre familias sólo para ALT $(p \leq 0.001)$ y no asi en DAP y $\mathrm{D}^{2} \mathrm{H}$. 
Cuadro $\mathrm{N}^{\circ} 10$.

ORDENAMIENTO DE LAS 30 MEJORES FAMILIAS SEGÚN D² H AJUSTADO POR BLOQUE Y PROCEDENCIA PARA EL ENSAYO DE LA PAILA.

\begin{tabular}{|c|c|c|c|c|c|c|}
\hline ORD & PROCEDENCIA & UBIC. & $\begin{array}{c}\text { FAMIL. } \\
\mathrm{N}^{\circ}\end{array}$ & $\begin{array}{l}\text { ALTURA } \\
\text { (m) }\end{array}$ & $\begin{array}{l}\text { DAP } \\
(\mathrm{cm})\end{array}$ & $\begin{array}{l}D^{2} \mathrm{H} \\
\left(m^{3}\right)\end{array}$ \\
\hline 1 & E. Lake Albacutya & VIC & 60 & 2,93 & 2,98 & 0,0037 \\
\hline 2 & Lake Hindmarsh & VIC & 26 & 2,64 & 2,81 & 0.0036 \\
\hline 3 & E. Lake Albacutya & VIC & 58 & 2,75 & 2,93 & 0,0034 \\
\hline 4 & E. Lake Albacutya & VIC & 61 & 2,97 & 2,87 & 0,0034 \\
\hline 5 & N. Lake Albacutya & VIC & 69 & 2,88 & 2,99 & 0,0033 \\
\hline 6 & Outlet Creek Rainbow & VIC & 33 & 2,71 & 2,82 & 0,0032 \\
\hline 7 & Lake Hindmarsh & VIC & 24 & 2,76 & 2,78 & 0,0030 \\
\hline 8 & Wimmera R/Glenorchi & VIC & 10 & 2,67 & 2,77 & 0,0030 \\
\hline 9 & Lake Hindmarsh & VIC & 23 & 2,90 & 2,81 & 0,0029 \\
\hline 10 & Wimmera R/Glenorchi & VIC & 13 & 2,87 & 2,83 & 0,0029 \\
\hline 11 & Wimmera R/Glenorchi & VIC & 11 & 2,82 & 2,66 & 0,0029 \\
\hline 12 & Wimmera R/Elmhurst & VIC & 7 & 2,54 & 2,69 & 0,0025 \\
\hline 13 & S. Lake Albacutya & VIC & 49 & 2.56 & 2,76 & 0.0025 \\
\hline 14 & Outlet Creek Rainbow & VIC & 40 & 2,64 & 2,68 & 0,0025 \\
\hline 15 & Wimmera R/Elmhurst & VIC & 4 & 2,84 & 2,83 & 0,0025 \\
\hline 16 & N. Lake Albacutya & VIC & 73 & 2,72 & 2,65 & 0,0025 \\
\hline 17 & N. Lake Albacutya & VIC & 68 & 2,58 & 2,64 & 0.0025 \\
\hline 18 & Wimmera R/Elmhurst & VIC & 5 & 2,67 & 2,65 & 0,0025 \\
\hline 19 & Outlet Creek Rainbow & VIC & 39 & 2.60 & 2,61 & 0,0025 \\
\hline 20 & S. Lake Albacutya & VIC & 53 & 2,68 & 2,65 & 0,0024 \\
\hline 21 & Lowan Valley-Saline & VIC & 81 & 2.70 & 2,56 & 0.0023 \\
\hline 22 & Wimmera R/Elmhrst & VIC & 1 & 2,45 & 2,48 & 0,0023 \\
\hline 23 & Wimmera R/Glenorchi & VIC & 19 & 2,46 & 2,78 & 0.0023 \\
\hline 24 & N. Lake Albacutya & VIC & 70 & 2.54 & 2,57 & 0,0023 \\
\hline 25 & E. Lake Albacutya & VIC & 62 & 2,52 & 2,27 & 0.0022 \\
\hline 26 & Outlet Creek Rainbow & VIC & 38 & 2.64 & 2,53 & 0,0022 \\
\hline 27 & Lake Hindmarsh & VIC & 22 & 2.61 & 2,36 & 0,0020 \\
\hline 28 & S. Lake Albacutya & VIC & 51 & 2,64 & 2,55 & 0,0020 \\
\hline 29 & S. Lake Albacutya & VIC & 54 & 2,45 & 2,57 & 0.0020 \\
\hline \multirow[t]{2}{*}{30} & Wimmera R/Elmhurst & VIC & 6 & 2,54 & 2,27 & 0,0020 \\
\hline & PROMEDIO & & & 2.68 & 2,68 & 0,0027 \\
\hline
\end{tabular}

El rango que existe entre las 10 familias de mejor y peor crecimiento se entrega en la Figura $\mathrm{N}^{\circ} 4$. No se presentaron diferencias significativas entre familias $(p>0,05)$ fluctuando los $\mathrm{D}^{2} \mathrm{H}$ entre 0.037 y $0.009 \mathrm{~m}^{3}$. lo que significa una superioridad sobre el $268 \%$ de la mejor familia respecto de la familia con menor crecimiento. También se observa que las mismas procedencias poseen familias entre los mayores y menores crecimientos, por ejemplo: entre las 10 familias de mayor crecimiento existen $\mathbf{4}$ familias de Lake Albacutya $(60,58,61,69)$, en cambio $6(55,66,63,47,78,56)$ de las 10 peores familias son de la misma procedencia. Sin embargo, el comportamiento de las familias procedentes de Lake Hindmarsh en este ensayo determinan 3 familias entre las 10 mejores $(26,24,23)$ y el resto de las 10 familias a probar, se mantienen alrededor del promedio para $\mathrm{D}^{2} \mathrm{H}$. 


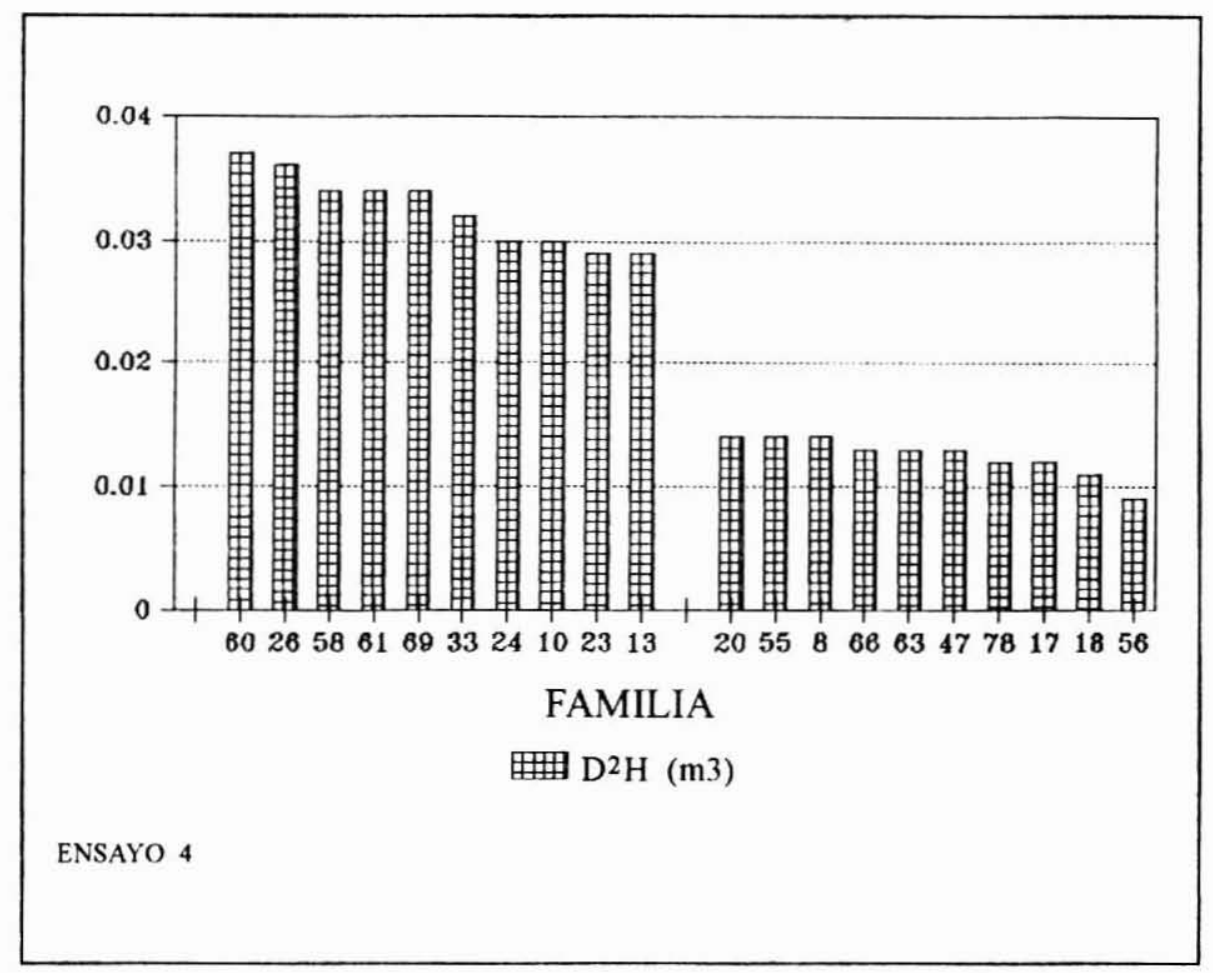

Figura $N^{\circ} 4$. E. Calmaldulensis - LA PAILA MEJORFS Y PEORES 10 FAMILIAS SEGÚN D² H

Antecedentes en la literatura muestran que el E. camaldulensis en condiciones de suelo y clima más favorables alcanzan niveles de crecimiento y adaptabilidad bastante superiores. a la misma edad, a los obtenidos en estos ensayos, asi por ejemplo, en una evaluación de ensayos de procedencias en dos comunas de Karnataka (Kalanakatte y Devbal) realizada por Chandra et al. (1994). en la India. donde sólo 2 procedencias fueron semejante a los ensayos nacionales: Emu Crek Petford (QLD) y Katherine (NT), las cuales alcanzan altura a los 4 años de 8.7 y $8.2 \mathrm{~m}$ en Kalanakatte y de 6,2 y $5,6 \mathrm{~m}$ en Devbal, respectivamente.

\section{Parámetros Genéticos.}

Los parámetros genéticos estimados en las poblaciones de E. camaldulensis dentro de cada ensayo son: heredabilidad individual $\left(h^{2}\right)$ y correlación genética. Además se entregan las correlaciones fenotipicas entre las variables estudiadas. 
En el Cuadro $\mathrm{N}^{\circ} 12$ se entregan las heredabilidades individuales y sus errores estandar para las variables ALT, DAC, DAP y $D^{2} \mathrm{H}$, por ensayo (Becker, 1984). La heredabilidad promedio ponderada y sus errores estandares se calcularon según el método de Cunningham et al. (1977).

Las heredabilidades calculadas en general son bajas, determinando un promedio entre los 4 ensayos de $0,19 \pm 0,02 ; 0,14 \pm 0,02$ y $0,12 \pm 0,01$, para la ALT, DAP (DAC) y $\mathrm{D}^{2} \mathrm{H}$, respectivamente (Cuadro $\mathrm{N}^{\circ} 12$ ). Estimaciones a nivel nacional en E. globulus ssp. globulus realizadas por Prado y Alvear (1993), para las mismas características de crecimiento a los 4 años de edad, entregan valores promedios de 3 ensayos de progenieprocedencia parecidos para la ALT $(0,20 \pm 0,02)$ y algo superiores para DAP $(0,21 \pm$ $0,02)$ y $D^{2} H(0,15 \pm 0,02)$. Los mayores valores de $h^{2}$ para las tres características evaluadas fueron obtenidos en el ensayo Tantehue, como consecuencia de la menor varianza ambiental determinada por la homogeneidad del sitio. situación que se hace muy evidente al comparar la $\mathrm{h}^{2}$ del DAP entre ensayos semejantes (Tantehue y La Paila). Sin duda que los bajos valores calculados en este estudio responden a la variabilidad encontrada en algunos de los ensayos.

Sin embargo. sería necesario volver sobre estos cálculos a una edad más avanzada. Por el momento, las heredabilidades calculadas para altura y diámetro, sugieren que ganancias genéticas razonables podrian lograrse mediante selección individual, considerando sólo al ensayo Tantehue (3). que posee heredabilidades muy superiores al resto de los ensayos para las tres caracteristicas de crecimiento, situación determinada en gran medida por la mejor calidad de sitio que permite una expresión precoz del potencial genético entre los hermanos (familias), potencial que es dificil de manifestar en sitios más pobres. La componente ambiental, en general hasta esta edad, resulta aún muy fuerte y determinante en el crecimiento de los árboles especialmente cuando están sometidos a condiciones de manejo deficientes, como lo ocurrido en los ensayos de Longotoma y La Paila. cuyas estimaciones de $\mathrm{h}^{2}$ fueron inferiores al resto de los ensayos debido a un aumento relativo de las varianzas bloque $\mathrm{e}$ interacción bloque $\mathrm{x}$ familia, las que determinan un aumento de la varianza fenotipica.

La correlación genética de cada ensayos y el promedio entre los ensayos se entregan en el Cuadro $\mathrm{N}^{\circ} 13$ y las correlaciones fenotipicas en el Cuadro 14. En general las correlaciones genéticas fueron mayores a las fenotípicas.

Las correlaciones genéticas y fenotipicas son bastantes altas entre las variables análizadas, lo que implica un alto componente genético responsable de la asociación entre estas caracteristicas de crecimiento (Cuadros $\mathrm{N}^{\circ} 13$ y $\mathrm{N}^{\circ} 14$ ), así en promedio la correlación genética entre ALT y DAP fue $0.90 \pm 0.02$, entre ALT y ${ }^{2} \mathrm{H}$ fue $0,94 \pm$ 0,01 y entre DAP y $D^{2} \mathrm{H}$ fue de $0,99 \pm 0,01$. En cambio las correlaciones fenotípicas promedio entre estas mismas características de crecimiento fueron: ALT-DAP $=0,75$; ALT $-D^{2} \mathrm{H}=0.79$ y DAP-D ${ }^{2} \mathrm{H}=0.90$ 


\section{Cuadro $\mathrm{N}^{\circ} 12$.}

HEREDABILIDAD Y ERROR ESTANDAR DE LOS PARÁMETROS DASOMÉTRICOS EN LOS DIFERENTES ENSAYOS DE $E$. camaldulensis.

\begin{tabular}{|c|c|c|c|c|}
\hline ENSAYOS & $\begin{array}{c}\text { ALTURA } \\
\mathrm{h}^{2} \pm \mathrm{se}\end{array}$ & $\begin{array}{c}\text { DAP } \\
h^{2} \pm s e\end{array}$ & \multicolumn{2}{|c|}{$\begin{array}{c}\mathrm{D}^{2} \mathrm{H} \\
\mathrm{h}^{2} \pm \mathrm{se}\end{array}$} \\
\hline MEL-MEL (44 meses) & $0,18 \quad 0,03$ & $0,13 \quad 0,03$ & 0,11 & 0,03 \\
\hline LONGOTOMA (44 meses) & $0,18 \quad 0,03$ & 0,12 & 0,07 & 0,01 \\
\hline TANTEHUE (42 meses) & $0,23 \quad 0,05$ & 0.27 & 0,17 & 0,01 \\
\hline LA PAILA (42 meses) & $0,19 \quad 0,06$ & 0,12 & 0,12 & 0,04 \\
\hline PROMEDIO & $0,19 \quad 0.02$ & 0.14 & 0,12 & 0,01 \\
\hline
\end{tabular}

- : DAC en los ensayos 1 y 2.

**: DACxALT en los ensayos 1 y 2.

Cuadro $\mathrm{N}^{\circ} 13$.

CORRELACIÓN GENÉTICA Y ERROR ESTANDAR DE LOS PARÁMETROS DASOMÉTRICOS EN LOS DIFERENTES ENSAYOS DE $E$. camaldulensis.

\begin{tabular}{|c|c|c|c|c|}
\hline ENSAYOS & $\begin{array}{c}\text { ALT-DAP* } \\
r_{G} \pm s e\end{array}$ & $\begin{array}{c}\text { ALT-D' }{ }^{2} \mathrm{H} \\
\mathrm{r}_{0} \pm \mathrm{se}\end{array}$ & \multicolumn{2}{|c|}{$\begin{array}{c}\text { DAP* }-D^{2} H^{* *} \\
r_{\sigma} \pm s e\end{array}$} \\
\hline MEL-MEL (44 meses) & $0,66 \quad 0,08$ & $\begin{array}{ll}0,91 & 0,03\end{array}$ & 0,91 & 0,03 \\
\hline LONGOTOMA (44 meses) & $0,71 \quad 0,07$ & 0,94 & 0,97 & 0,01 \\
\hline TANTEHUE (42 meses) & $0.83 \quad 0,04$ & 0,91 & 0,99 & 0,01 \\
\hline LA PAILA ( 42 meses) & $0.95 \quad 0.02$ & 0,97 & 0,99 & 0,01 \\
\hline PROMEDIO & 0.02 & 0.94 & 0,99 & 0,01 \\
\hline
\end{tabular}

* : DAC en los ensayos 1 y 2.

**: DAC ${ }^{2} x A L T$ en los ensayos 1 y 2.

Cuadro $\mathrm{N}^{\circ} 14$.

CORRELACIÓN FENOTIPICA DE LOS PARAMMETROS DASOMÉTRICOS EN LOS DIFERENTES ENSAYOS DE E. camaldulensis.

\begin{tabular}{|c|c|c|c|}
\hline ENSAYOS & $\begin{array}{c}\text { ALT-DAP* } \\
r_{\mathrm{P}}\end{array}$ & $\begin{array}{c}\text { ALT-D'D } \mathrm{H}^{* *} \\
\mathrm{r}_{\mathrm{p}}\end{array}$ & $\begin{array}{c}\mathrm{DAP}^{*}-\mathrm{D}^{2} \mathrm{H}^{* *} \\
\mathrm{r}_{\mathrm{p}}\end{array}$ \\
\hline MEL-MEL (44 meses) & 0.66 & 0,76 & 0,90 \\
\hline LONGOTOMA (44 meses) & 0,83 & 0,84 & 0,89 \\
\hline TANTEHUE (42 meses) & 0.78 & 0,84 & 0,90 \\
\hline LA PAIL.A (42 meses) & 0.74 & 0,71 & 0,92 \\
\hline PROMEDIO & 0.75 & 0,79 & 0,90 \\
\hline
\end{tabular}

$\because$ DAC en los ensayos 1 y 2.

**: DAC ${ }^{2} x A L T$ en los ensayos 1 y 2.

La correlación genética y fenotípica estimada entre ALT y DAP presentó una menor relación y con mayor variación en los ensayos con procedencias de diferente origen, 
Mel-Mel (1) y Longotoma (2) determinado principalmente por relaciones distintas entre las procedencias de VIC y NSW.

\section{CONCLUSIONES.}

Los resultados obtenidos para el crecimiento son consistentes con evaluaciones efectuadas en los mismos ensayos a la edad de 24 meses (Gutiérrez y Chung, 1993).

En general, a excepción del ensayo La Paila, se observan niveles altos de sobrevivencia. Sin embargo, las mayores mortalidades fueron observadas en los testigos nacionales, salvo en el ensayo Tantehue.

Se aprecia una clara superioridad en el crecimiento manifestado por las procedencias australianas en relación a las nacionales utilizadas como testigos.

Las cifras que se entregan revelan importantes diferencias en el desarrollo de la especie en las distintas localidades donde fue ensayada. Las razones para estas diferencias están dadas tanto por variaciones en las condiciones de clima y suelo, como por variaciones en la topografia y exposición de los ensayos. En el caso del ensayo Tantehue el mayor crecimiento se debe, sin duda, a la calidad del suelo y a la exposición más humeda al momemto del establecimiento; en cambio, en La Paila el menor desarrollo responde a las condiciones climáticas y topografia del suelo imperantes en el área.

A pesar de que es posible identificar procedencias que presentan un buen crecimiento promedio, en la mayoria de los casos la variación que exhiben sus progenies es tan amplia que impide generalizar juicios sobre las procedencias completas. Análogamente, procedencias que en promedio no exhiben crecimientos satisfactorios, presentan progenies que alcanzan valores de gran interés.

Al comparar el desarrollo de los árboles en cada uno de los 4 lugares de ensayo. se encuentran diferencias estadísticamente significativas entre las procedencias, cualquicra sea la variable considerada (ALT, DAP o $D^{2} \mathrm{H}$ ). En todos los casos las mejores procedencias corresponden a Lake Albacutya y Lake Hindmarsh de Victoria.

En el caso del ensayo La Paila se producen diferencias significativas en el desarrollo y la sobrevivencia. fundamentalemente debido al sitio de muy baja calidad. Esto deja de manifiesto la diferencia en adaptabilidad de las procedencias de Victoria.

Es categórico el bajo crecimiento de la procedencia Avon River-Navarre de Victoria que en todos los ensayos presentó un crecimiento deficiente, situación no muy evidente a edad más temprana. Por el contrario. la procedencia Umberumberka Creek (NSW) continúa con el menor desarrollo demostrado ya a los dos años de edad (Gutiérrez y Chung. 1993) 
En general, las progenies que exhiben los mejores crecimientos corresponden a las procedencias de los lagos Hindmarsh y Albacutya. Una situación similar se observa en las progenies de la procedencia Outlet Creek Rainbow

Los parámetros genéticos calculados, indican que podría esperarse una moderada ganancia a partir de la selección de árboles individuales, dentro de las mejores familias. Por lo tanto, a esta edad el método de mejoramiento genético más indicado sería en base a selección familiar incluyendo varias características en un índice de selección.

La base de un proyecto de mejoramiento genético de mediano plazo deberá estar constituida, principalmente, por familias procedentes de Victoria. Sin embargo, no debe descartarse el aporte de caracteristicas positivas, ya sea en la forma. calidad de la. madera o resistencia a plagas y enfermedades de las otras procedencias.

\section{REFERENCIAS.}

Barros, S., 1990. Ensayos de Procedencias de Eucalyptus camaldulensis Dehn en la Zona Semiárida de Chile. Ciencia e Investigación Forestal, 4(2): 171-182.

Becker, W.A., 1984. Manual of Quantitative Genetics. Academic Enterprises. Pullman. USA. $186 \mathrm{p}$.

Chandra, B. K. J.; Kariyappa, G.S. and Manjunath, B.E., 1994. Evaluation of Eucalyptus camaldulensis Provenance Trials in Kamataka. Indian Forester, 120 (8): 670 - 676.

Cunningham, E.P, T. O'Byrne and A. A. Mescal., 1977. Genetic Relationship between Beef and Dairy Traits in Friesian cattle. Irish J. Agric. Res., 16: 243-249.

Gutiérrez, B. y Chung, P., 1993. Crecimiento Inicial de 23 Procedencias y 196 Familias de Eucalyptus camaldulensis Denh en Cuatro Sitios de la Zona Central de Chile. Ciencia e Investigación Forestal, 7(1): 5-21

Infante, P. y Prado, J., 1989. Crecimiento de 35 Procedencias de Eucalyptus globulus ssp. globulus en Etapa de Vivero. Ciencia e Investigación Forestal, 2(7): 83 - 92.

Infante, P. y Prado, J., 1991. Crecimiento Juvenil de 32 Procedencias y 203 Familias de Eucalyptus globulus ssp. globulus en la Zona Costera de la VIII Región de Chile. Ciencia e Investigacion Forestal, 5(2):251 - 265

INFOR., 1986. Especies Forestales Exóticas de Interés Económico para Chile. Santiago, Chile. Gerencia de Desarrollo, CORFO AF 86/32.167 p.

Prado, J.A. y Alvear, C., 1993. Resultados de Ensayos de Procedencias y Progenies de Eucalyptus globulus en la Zona Centro-Sur de Chile. Actas: Simposio: Los Eucaliptos en el Desarrollo Forestal de Chile Pucón-Chile 24-26 Noviembre, 61 - 80 .

Whitemann, P.H.; Dean, C.A.; Doran, J.C. and Cameron, J.N., 1992 Genetic Parameters and Selection Strategies for Eucalyptus nitens (Dean and Maiden) in Victoria. Silvae Genetica, 41: $77-81$ 